\title{
On approximation to real numbers by algebraic numbers
}

\author{
by
}

\section{K. I. TishChenko (Minsk)}

1. Introduction. In this paper we study the problem of solvability of the inequality

$$
|\xi-\alpha|<c(\xi, n) H(\alpha)^{-A}
$$

in algebraic numbers $\alpha$ of degree $\leq n$, where $A>0, \xi$ is a real number which is not an algebraic number of degree $\leq n, H(\alpha)$ is the height of $\alpha$. In 1842 Dirichlet proved that for any real number $\xi$ there exist infinitely many rational numbers $p / q$ such that $|\xi-p / q|<q^{-2}$. In $1961 \mathrm{E}$. Wirsing [9] proved that (1.1) has infinitely many solutions if $A=n / 2+\gamma_{n}$, where $\lim _{n \rightarrow \infty} \gamma_{n}=$ 2. Moreover, he conjectured that the inequality (1.1) has infinitely many solutions if $A=n+1-\varepsilon$, where $\varepsilon>0$. Further it has been conjectured [5] that the exponent $n+1-\varepsilon$ can be replaced even by $n+1$. This problem has not been solved except in some special cases. In 1965 V. G. Sprindžuk [6] proved that the Conjecture of Wirsing holds for almost all real numbers. In 1967 H. Davenport and W. Schmidt [3] obtained new results in the theory of linear forms. These enabled them to prove the Conjecture for $n=2$. In 1993 [1] the following improvement of the Theorem of Wirsing was obtained: $A=n / 2+\gamma_{n}^{\prime}$, where $\lim _{n \rightarrow \infty} \gamma_{n}^{\prime}=3$. In 1992-1997 a new method was introduced, improving the Theorem of Wirsing for $n \leq 10([7,8])$.

In this paper we prove the following

TheOREM. For any real number $\xi$ which is not an algebraic number of degree $\leq n$, there exist infinitely many algebraic numbers $\alpha$ of degree $\leq n$ such that

$$
|\xi-\alpha| \ll H(\alpha)^{-A} .
$$

Here and below $3 \leq n \leq 7$, « is the Vinogradov symbol, and $A=A(n)$ is the positive root of the quadratic equation

$$
(3 n-5) X^{2}-\left(2 n^{2}+n-9\right) X-n-3=0 .
$$

The implicit constant in $\ll$ depends on $\xi$ and $n$ only.

2000 Mathematics Subject Classification: Primary 11J68. 
The following table contains the values of $A$ corresponding to Wirsing's Theorem, the Theorem above and the Conjecture:

\begin{tabular}{cccc}
\hline$n$ & Wirsing, 1961 & Theorem & Conj. \\
\hline 3 & 3.2807764 & 3.4364917 & 4 \\
4 & 3.8228757 & 4.1009866 & 5 \\
5 & 4.3507811 & 4.7677925 & 6 \\
6 & 4.8708287 & 5.4350702 & 7 \\
7 & 5.3860009 & 6.1024184 & 8 \\
\hline
\end{tabular}

2. Preliminaries. We can confine ourselves to the range $0<\xi<1 / 4$. We suppose that there exists a real number $0<\xi<1 / 4$ which is not an algebraic number of degree $\leq n$, such that

$$
\forall c>0 \exists \widetilde{H}_{0}>0 \forall \alpha \in \mathbb{A}_{n}, H(\alpha)>\widetilde{H}_{0}, \quad|\xi-\alpha|>c H(\alpha)^{-A},
$$

where $\mathbb{A}_{n}$ denotes the set of algebraic numbers of degree $\leq n$. Also, we may assume that $\widetilde{H}_{0}>((2 n) !)^{30 n} e^{60 n^{2}}$.

By Lemma 1 of [2] we have

$$
|\xi-\alpha| \leq n \frac{|P(\xi)|}{\left|P^{\prime}(\xi)\right|}
$$

where $\alpha$ is the root of the polynomial $P(x)$ closest to $\xi$. In fact, we get

$$
\frac{\left|P^{\prime}(\xi)\right|}{|P(\xi)|}=\left|\sum_{i=1}^{n} \frac{1}{\xi-\alpha_{i}}\right| \leq \sum_{i=1}^{n} \frac{1}{\left|\xi-\alpha_{i}\right|} \leq \frac{n}{|\xi-\alpha|}
$$

which gives (2.2). Put

$$
c_{T}=4^{n^{2}}(n !)^{4 n^{3}} \xi^{-2 n^{5}} .
$$

By (2.1) and (2.2) we obtain

$$
\begin{gathered}
\exists \widetilde{H}_{0}>0 \forall Q(x) \in \mathbb{Z}[x], \operatorname{deg} Q(x) \leq n,|Q|>\widetilde{H}_{0}, \\
\frac{|Q(\xi)|}{\left|Q^{\prime}(\xi)\right|}>c_{T}|Q|^{-A} .
\end{gathered}
$$

Throughout the paper $|L|$ denotes the height of the polynomial $L(x)$.

\section{Auxiliary lemmas}

LEMMA 3.1. Let $L(x)=c_{n} x^{n}+\ldots+c_{1} x+c_{0}$ be a polynomial with integer coefficients such that $|L(\xi)|<1 / 2$. Then there is an index $j_{1} \in\{1, \ldots, n\}$ such that $\left|c_{j_{1}}\right|=|L|$. 
Proof. Assume that $\left|c_{j_{1}}\right|<|L|$ for any $j_{1} \in\{1, \ldots, n\}$. Then

$$
|L(\xi)|=\left|\sum_{\nu=0}^{n} c_{\nu} \xi^{\nu}\right|>\left|-\sum_{\nu=1}^{n}\right| L\left|\xi^{\nu}+\right| L||=|L|\left|-\sum_{\nu=1}^{n} \xi^{\nu}+1\right|>\frac{1}{2} .
$$

LEMMA 3.2. Let $L(x)$ be a polynomial and $j_{1}$ an index as in Lemma 3.1. Suppose $\left|c_{i}\right| \leq \xi^{n-1}|L|$ for every $i \in\{1, \ldots, n\} \backslash\left\{j_{1}\right\}$. Then $|L|<\xi^{-n+1}\left|L^{\prime}(\xi)\right|$.

Proof. We have

$$
\left|L^{\prime}(\xi)\right|=\left|\sum_{\nu=1}^{n} \nu c_{\nu} \xi^{\nu-1}\right|=\left|j_{1} c_{j_{1}} \xi^{j_{1}-1}+\left(\sum_{\nu=1}^{n} \nu c_{\nu} \xi^{\nu-1}-j_{1} c_{j_{1}} \xi^{j_{1}-1}\right)\right| .
$$

Since $\left|j_{1} c_{j_{1}} \xi^{j_{1}-1}\right|=j_{1}|\bar{L}| \xi^{j_{1}-1} \geq n|L| \xi^{n-1}$,

$$
\begin{aligned}
\left|\sum_{\nu=1}^{n} \nu c_{\nu} \xi^{\nu-1}-j_{1} c_{j_{1}} \xi^{j_{1}-1}\right| & \leq \xi^{n-1}|L|\left(\sum_{\nu=1}^{n} \nu \xi^{\nu-1}-j_{1} \xi^{j_{1}-1}\right) \\
& \leq \xi^{n-1}|L| \sum_{\nu=1}^{n-1} \nu \xi^{\nu-1}
\end{aligned}
$$

and $n-\sum_{\nu=1}^{n-1} \nu \xi^{\nu-1}>1$, we obtain

$$
\begin{aligned}
\left|L^{\prime}(\xi)\right| & \geq\left|j_{1} c_{j_{1}} \xi^{j_{1}-1}\right|-\left|\sum_{\nu=1}^{n} \nu c_{\nu} \xi^{\nu-1}-j_{1} c_{j_{1}} \xi^{j_{1}-1}\right| \\
& \geq n \xi^{n-1}|L|-\xi^{n-1}|L| \sum_{\nu=1}^{n-1} \nu \xi^{\nu-1}=\xi^{n-1}|L|\left(n-\sum_{\nu=1}^{n-1} \nu \xi^{\nu-1}\right) \\
& >\xi^{n-1}|L| .
\end{aligned}
$$

Notations. In this section $L^{(k)}(x)$ denotes the $k$ th derivative of a polynomial $L(x)$. However, in Sections $4-7$ we will use $\widetilde{Q}_{i}^{(l)}(x)$ to denote the polynomial with indices $l$ and $i$.

LEMma 3.3. For any polynomials $F(x)$ and $G(x)$ the following identity is valid:

$$
\left.R(F, G) \equiv\left|\begin{array}{ccccc}
\frac{F^{(l)}(\xi)}{l !} & \ldots & F^{\prime}(\xi) & F(\xi) & \\
\ddots & & \ddots & \ddots & \\
& \frac{F^{(l)}(\xi)}{l !} & \ldots & F^{\prime}(\xi) & F(\xi)
\end{array}\right|\right\} m,
$$


where $R(F, G)$ denotes the resultant of $F(x)$ and $G(x), \xi$ is any real, complex or p-adic number, $\operatorname{deg} F(x)=l, \operatorname{deg} G(x)=m$.

Proof. Write

$$
\begin{array}{cc}
F(x)=\sum_{\nu=0}^{l} a_{\nu} x^{\nu}=a_{l} \prod_{\nu=1}^{l}\left(x-\alpha_{\nu}\right), & G(x)=\sum_{\nu=0}^{m} b_{\nu} x^{\nu}=b_{m} \prod_{\nu=1}^{m}\left(x-\beta_{\nu}\right), \\
\widetilde{F}(x)=F(x+\xi)=\sum_{\nu=0}^{l} \widetilde{a}_{\nu} x^{\nu}, & \widetilde{G}(x)=G(x+\xi)=\sum_{\nu=0}^{m} \widetilde{b}_{\nu} x^{\nu} .
\end{array}
$$

Denote by $\Delta_{l, m}\left(A_{i}, B_{j}\right)$ the determinant obtained from (3.1) by replacing $F^{(i)}(\xi) / i$ ! and $G^{(j)}(\xi) / j$ ! with $A_{i}$ and $B_{j}, 0 \leq i \leq l, 0 \leq j \leq m$, respectively. For example, according to the definition of resultant we have $R(F, G)=$ $\Delta_{l, m}\left(a_{i}, b_{j}\right)$. We now obtain

$$
\begin{aligned}
R(F, G) & =a_{l}^{m} b_{m}^{l} \prod_{i, j}\left(\alpha_{i}-\beta_{j}\right)=a_{l}^{m} b_{m}^{l} \prod_{i, j}\left(\alpha_{i}-\xi-\left(\beta_{j}-\xi\right)\right)=\Delta_{l, m}\left(\widetilde{a}_{i}, \widetilde{b}_{j}\right) \\
& =\Delta_{l, m}\left(\frac{\widetilde{F}^{(i)}(0)}{i !}, \frac{\widetilde{G}^{(j)}(0)}{j !}\right)=\Delta_{l, m}\left(\frac{F^{(i)}(\xi)}{i !}, \frac{G^{(j)}(\xi)}{j !}\right) .
\end{aligned}
$$

Lemma 3.4. Let $F(x), G(x) \in \mathbb{Z}[x]$ be nonzero polynomials with $\operatorname{deg} F(x)$ $=l \leq n, \operatorname{deg} G(x)=m \leq n, l m \geq 2$. Suppose that $F(x)$ and $G(x)$ have no common root. Then at least one of the following estimates is true:

(I) $1<c_{R} \max (|F(\xi)|,|G(\xi)|)^{2} \max (|F|,|G|)^{m+l-2}$,

(II) $1<c_{R} \max \left(|F(\xi)| \cdot\left|F^{\prime}(\xi)\right| \cdot\left|G^{\prime}(\xi)\right|,|G(\xi)| \cdot\left|F^{\prime}(\xi)\right|^{2}\right)|F|^{m-2} \mid G^{l-1}$,

(III) $1<c_{R} \max \left(|G(\xi)| \cdot\left|F^{\prime}(\xi)\right| \cdot\left|G^{\prime}(\xi)\right|,|F(\xi)| \cdot\left|G^{\prime}(\xi)\right|^{2}\right)|F|^{m-1} \widehat{G}^{l-2}$, where $0<\xi<1$ and $c_{R}=(2 n) !((n+1) !)^{2 n-2}$.

Proof. Consider the identity of Lemma 3.3. Since the polynomials $F(x), G(x) \in \mathbb{Z}[x]$ have no common root, it follows that

$$
|R(F, G)| \geq 1 \text {. }
$$

We will obtain an upper bound for the absolute value of the determinant (3.1). Let us expand it with respect to the last column. Obviously, any nonzero term contains the factor $F(\xi)$ or $G(\xi)$. We distinguish two cases.

CASE A. Suppose that some nonzero term contains $F(\xi)^{2}, G(\xi)^{2}$ or $F(\xi) G(\xi)$. Using the inequality

$$
\left|L^{(k)}(\xi)\right|<(n+1) !|L|
$$

where $\operatorname{deg} L(x) \leq n$, we estimate other factors. Hence this term has absolute value at most

$$
((n+1) !)^{m+l-2} \max (|F(\xi)|,|G(\xi)|)^{2} \max (|F|,|G|)^{m+l-2} .
$$


CASE B. Suppose that some nonzero term contains $F(\xi)$ or $G(\xi)$ together with the other factors $F^{(i)}(\xi) / i$ ! or $G^{(j)}(\xi) / j$ ! where $1 \leq i \leq l, 1 \leq j \leq m$. If we expand the determinant (3.1) according to the last three columns, we see that the term considered contains one of the following expressions: $F(\xi) F^{\prime}(\xi) G^{\prime}(\xi), G(\xi) F^{\prime}(\xi)^{2}, G(\xi) F^{\prime}(\xi) G^{\prime}(\xi)$ or $F(\xi) G^{\prime}(\xi)^{2}$. Using (3.4) we conclude that this term has absolute value at most

$$
((n+1) !)^{m+l-3} \max \left(|F(\xi)| \cdot\left|F^{\prime}(\xi)\right| \cdot\left|G^{\prime}(\xi)\right|,|G(\xi)| \cdot\left|F^{\prime}(\xi)\right|^{2}\right)|F|^{m-2}|G|^{l-1}
$$

or

$((n+1) !)^{m+l-3} \max \left(|G(\xi)| \cdot\left|F^{\prime}(\xi)\right| \cdot\left|G^{\prime}(\xi)\right|,|F(\xi)| \cdot\left|G^{\prime}(\xi)\right|^{2}\right)|F|^{m-1} \mid G^{l-2}$.

Finally, when expanding the determinant $(3.1)$, we obtain $(l+m)$ ! terms.

Combining this information with (3.3), we get (3.2)(I)-(III).

The following two lemmas are well known.

Lemma 3.5 (see [4], [5]). Let $R(x), R_{1}(x), \ldots, R_{\nu}(x)$ be polynomials such that $R(x)=R_{1}(x) \ldots R_{\nu}(x), \operatorname{deg} R(x)=l$. Then

$$
e^{-l}\left|R_{1} \ldots\right| R_{\nu}|\leq| R\left|\leq(l+1)^{\nu-1}\right| R_{1}|\ldots| \overrightarrow{R_{\nu}} .
$$

LEMMA 3.6. Let $F(x)$ and $G(x)$ be polynomials with integer coefficients of degree $\leq l$. Let $F(x)$ be a polynomial irreducible over $\mathbb{Z}$ with $|F|>e^{l}|G|$. Then $F(x)$ and $G(x)$ have no common root.

Proof. Assume that $F(x)$ and $G(x)$ have a common root. Then there exists a polynomial $\widetilde{F}(x) \in \mathbb{Z}[x], \widetilde{F}(x) \not \equiv 1$, dividing both $F(x)$ and $G(x)$. Since $F(x)$ is irreducible, we have $\widetilde{F}(x) \equiv F(x)$. Therefore $G(x)=F(x) \widetilde{G}(x)$, where $\widetilde{G}(x) \in \mathbb{Z}[x]$. By (3.5) we have $|G| \geq e^{-l}|F| \widetilde{G}\left|\geq e^{-l}\right| F$.

Lemma 3.7. Consider the following system of inequalities:

$$
\left\{\begin{array}{l}
\left|a_{11} x_{1}+\ldots+a_{1 n} x_{n}\right| \leq A_{1}, \\
\left|a_{21} x_{1}+\ldots+a_{2 n} x_{n}\right| \leq A_{2}, \\
\ldots \ldots \ldots \ldots \ldots \ldots \ldots \ldots \ldots \\
\left|a_{n 1} x_{1}+\ldots+a_{n n} x_{n}\right| \leq A_{n},
\end{array}\right.
$$

where $a_{i j} \in \mathbb{R}, A_{i} \in \mathbb{R}^{+}, 1 \leq i, j \leq n$. Suppose that

(I) for any $1 \leq j \leq n, \max _{2 \leq i \leq n}\left(\left|a_{i j}\right|\right) \leq B_{j}, \min _{1 \leq j \leq n-1}\left(B_{j}\right) \geq B_{n}>0$;

(II) $\max _{1 \leq j \leq n-1}\left(\left|a_{1 j}\right|\right) \leq\left|a_{1 n}\right|, a_{1 n} \neq 0$;

(III) $\max _{2 \leq \nu \leq n-1}\left(A_{\nu}\right) \leq A_{n}$;

(IV) $|\Delta|>c_{d}\left|a_{1 n}\right| B_{1} \ldots B_{n-1}$, where $\Delta$ is the determinant of the system (3.6), and $c_{d}$ is some positive constant.

Then for any solution $\left(\widetilde{x}_{1}, \ldots, \widetilde{x}_{n}\right) \in \mathbb{R}^{n}$ of the system (3.6) the following estimates hold:

$$
\left|\widetilde{x}_{l}\right|<\frac{n !}{c_{d}} B_{l}^{-1} \max \left(\frac{A_{1} B_{n}}{\left|a_{1 n}\right|}, A_{n}\right) \quad(1 \leq l \leq n) .
$$


Pr o of. Using the Theorem of Cramer, we have

$$
\left|\widetilde{x}_{l}\right|=\frac{\left|\Delta_{l}\right|}{|\Delta|} \quad(1 \leq l \leq n)
$$

where $\Delta_{l}$ is the determinant obtained from $\Delta$ by replacing $l$ th column with $\left[\theta_{1} A_{1}, \ldots, \theta_{n} A_{n}\right]^{\mathrm{T}},\left|\theta_{\nu}\right| \leq 1,1 \leq \nu \leq n$.

When expanding $\Delta_{l}$ with respect to the $l$ th column, we get

$$
\left|\Delta_{l}\right| \leq n \max \left(A_{1}\left|M_{1}\right|, \ldots, A_{n}\left|M_{n}\right|\right),
$$

where $M_{\nu}$ are the minors corresponding to $\theta_{\nu} A_{\nu}$ for $1 \leq \nu \leq n$.

By (I) we have

$$
\left|M_{1}\right| \leq(n-1) ! B_{1} \ldots B_{n} B_{l}^{-1} .
$$

Let us show that

$$
\left|M_{\nu}\right| \leq(n-1) !\left|a_{1 n}\right| B_{1} \ldots B_{n-1} B_{l}^{-1} \quad(2 \leq \nu \leq n) .
$$

In fact, by (II) the absolute values of $a_{1 j}$ from the first line of the minors $M_{\nu}, 2 \leq \nu \leq n$, are less than or equal to $\left|a_{1 n}\right|$. On the other hand, by (I) the absolute values of any minors $m_{\nu j}$ of $M_{\nu}$ which correspond to the elements $a_{1 j}$ are less than or equal to $(n-2) ! B_{1} \ldots B_{n-1} B_{l}^{-1}$. This gives (3.11).

Using (III) and (3.9)-(3.11), we get

$$
\left|\Delta_{l}\right| \leq n ! B_{1} \ldots B_{n-1} B_{l}^{-1} \max \left(A_{1} B_{n}, A_{n}\left|a_{1 n}\right|\right) .
$$

By substituting the estimate (IV) and (3.12) into (3.8), we obtain (3.7).

4. Construction of $\widetilde{Q}_{i}^{(0)}(x), \ldots, \widetilde{Q}_{i}^{(n-1)}(x)$. Fix some $h \in \mathbb{N}, h>\widetilde{H}_{0}$. We consider the finite set of polynomials $P(x) \in \mathbb{Z}[x]$ with $\operatorname{deg} P(x) \leq n$, $|P| \leq h$. Their values at $\xi$ are distinct. Hence we can choose a unique (up to sign) polynomial $\widetilde{P}_{0}(x) \in \mathbb{Z}[x], \widetilde{P}_{0}(x) \not \equiv 0$, with minimal absolute value at $\xi$.

Put

$$
c_{p}=n ! \xi^{-n^{2}} .
$$

We now increase $h$ until a polynomial $\widetilde{P}_{1}(x) \in \mathbb{Z}[x], \widetilde{P}_{1}(x) \not \equiv 0$, of degree $\leq n$ with $\left|\widetilde{P}_{1}\right| \leq h,\left|\widetilde{P}_{1}(\xi)\right|<c_{p}^{-1}\left|\widetilde{P}_{0}(\xi)\right|$ appears. If there are several polynomials of this kind, pick one with minimal absolute value at $\xi$. It is clear that $\widetilde{H}_{0}<\mid \widetilde{P}_{1}$. We increase $h$ again until a polynomial $\widetilde{P}_{2}(x) \in \mathbb{Z}[x]$ of degree $\leq n$ with $\widetilde{H}_{0}<\left|\widetilde{P}_{1}\right|<\left|\widetilde{P}_{2}\right| \leq h,\left|\widetilde{P}_{2}(\xi)\right|<c_{p}^{-1}\left|\widetilde{P}_{1}(\xi)\right|$ appears. By repeating this process, we obtain a sequence of polynomials $\widetilde{P}_{i}(x) \in \mathbb{Z}[x]$, 
$\operatorname{deg} \widetilde{P}_{i}(x) \leq n$, such that

(i) $1 / 2>\left|\widetilde{P}_{1}(\xi)\right|>c_{p}\left|\widetilde{P}_{2}(\xi)\right|>\ldots>c_{p}^{k-1}\left|\widetilde{P}_{k}(\xi)\right|>\ldots$,

(ii) $\widetilde{H}_{0}<\left|\widetilde{P}_{1}\right|<\left|\widetilde{P}_{2}\right|<\ldots<\left|\widetilde{P}_{k}\right|<\ldots$,

(iii) $\forall P(x) \in \mathbb{Z}[x], P(x) \not \equiv 0, \operatorname{deg} P(x) \leq n,|P|<\mid \widetilde{P}_{k+1}$,

$$
|P(\xi)| \geq c_{p}^{-1}\left|\widetilde{P}_{k}(\xi)\right| \text {. }
$$

For any natural $i$ we set

$$
\widetilde{Q}_{i}^{(0)}(x)=\widetilde{P}_{i}(x) .
$$

Write $\widetilde{Q}_{i}^{(0)}(x)=a_{n}^{(0)} x^{n}+\ldots+a_{1}^{(0)} x+a_{0}^{(0)}$. By Lemma 3.1 there is an index $j_{1} \in\{1, \ldots, n\}$ such that $\left|a_{j_{1}}^{(0)}\right|=\widetilde{Q}_{i}^{(0)} \mid$.

We successively construct nonzero polynomials $\widetilde{Q}_{i}^{(0)}(x), \ldots, \widetilde{Q}_{i}^{(n-1)}(x)$ in $\mathbb{Z}[x]$ of degree $\leq n$ and distinct integers $j_{1}, \ldots, j_{n}$ from $\{1, \ldots, n\}$. We write $\widetilde{Q}_{i}^{(l)}(x)=a_{n}^{(l)} x^{n}+\ldots+a_{1}^{(l)} x+a_{0}^{(l)}, 0 \leq l \leq n-1$. The polynomials $\widetilde{Q}_{i}^{(l)}(x)$ and the numbers $j_{l+1}$ (which we call the indices of the $\widetilde{Q}_{i}$-system) will have the following properties:

$\left(1_{l}\right) \quad\left|\widetilde{Q}_{i}^{(l)}(\xi)\right|<c_{p}^{-1}\left|\widetilde{P}_{i-1}(\xi)\right|$,

$\left(2_{l}\right) \quad\left|a_{j_{\mu}}^{(l)}\right| \leq c_{p}^{-1} \widetilde{\widetilde{Q}_{i}^{(\mu-1)}} \quad(\mu=1, \ldots, l)$,

$\left(3_{l}\right) \quad\left|a_{j_{l+1}}^{(l)}\right|>\xi^{n-1} \widetilde{\widetilde{Q}_{i}^{(l)}} \mid$

(if $l=0$, we have $\left(1_{l}\right),\left(3_{l}\right)$ only). Moreover, if for some $0 \leq l \leq n-1$ any nonzero polynomial $Q(x)=a_{n} x^{n}+\ldots+a_{1} x+a_{0} \in \mathbb{Z}[x]$ satisfies

$$
\begin{gathered}
|Q(\xi)|<c_{p}^{-1}\left|\widetilde{P}_{i-1}(\xi)\right|, \\
\left|a_{j_{\mu}}\right| \leq c_{p}^{-1} \sqrt[\widetilde{Q}_{i}^{(\mu-1)} \mid]{(\mu=1, \ldots, l)}
\end{gathered}
$$

(if $l=0$, we have $|Q(\xi)|<c_{p}^{-1}\left|\widetilde{P}_{i-1}(\xi)\right|$ only), then $|Q| \geq\left|\widetilde{Q}_{i}^{(l)}\right|$. In other words, $\widetilde{Q}_{i}^{(l)}(x)$ has minimum height among nonzero polynomials in $\mathbb{Z}[x]$ with $\left(1_{l}\right),\left(2_{l}\right)$. We call this the minimality property of $\widetilde{Q}_{i}^{(l)}(x), 0 \leq l \leq n-1$.

The pair $\left(\widetilde{Q}_{i}^{(0)}(x), j_{1}\right)$ has the desired properties. Suppose $0 \leq t<$ $n-1$, and $\left(\widetilde{Q}_{i}^{(0)}(x), j_{1}\right), \ldots,\left(\widetilde{Q}_{i}^{(t)}(x), j_{t+1}\right)$ have been constructed so that $\left(1_{l}\right),\left(2_{l}\right),\left(3_{l}\right)$ with $l=0, \ldots, t$ and the minimality property hold, and $j_{1}, \ldots$ $\ldots, j_{t+1}$ are distinct integers in $\{1, \ldots, n\}$. By Minkowski's Theorem on linear forms there is a nonzero polynomial $Q(x)=a_{n} x^{n}+\ldots+a_{1} x+a_{0} \in \mathbb{Z}[x]$ 
having

$$
\begin{aligned}
& |Q(\xi)|<c_{p}^{-1}\left|\widetilde{P}_{i-1}(\xi)\right|, \\
& \left|a_{j_{\mu}}\right| \leq c_{p}^{-1} \sqrt{\widetilde{Q}_{i}^{(\mu-1)} \mid} \quad(\mu=1, \ldots, t+1), \\
& \left|a_{k_{\eta}}\right| \leq\left(c_{p}^{-t-2}\left|\widetilde{P}_{i-1}(\xi)\right| \prod_{\nu=0}^{t}\left|\widetilde{Q}_{i}^{(\nu)}\right|\right)^{-1 /(n-t-1)} \quad(\eta=1, \ldots, n-t-1),
\end{aligned}
$$

where $\left\{k_{1}, \ldots, k_{n-t-1}\right\}=\{1, \ldots, n\} \backslash\left\{j_{1}, \ldots, j_{t+1}\right\}$.

If there are several polynomials of this kind, pick one whose height is minimal. We denote it by $\widetilde{Q}_{i}^{(t+1)}(x)$. By Lemma 3.1 , there is an index $j$ in $\{1, \ldots, n\}$ such that $\left|a_{j}^{(t+1)}\right|=\left|\widetilde{Q}_{i}^{(t+1)}\right|$. On the other hand, by the minimality property of $\widetilde{Q}_{i}^{(l)}(x)$ we have $\left|\widetilde{Q}_{i}^{(l)}\right| \leq \widetilde{Q}_{i}^{(t+1)}$ for any $0 \leq l \leq t$. Hence $\left|a_{j_{\mu}}^{(t+1)}\right|<\left|\widetilde{Q}_{i}^{(\mu-1)}\right| \leq \mid \widetilde{Q}_{i}^{(t+1)}$ for $\mu=1, \ldots, t+1$. Therefore $j$ is distinct from $j_{1}, \ldots, j_{t+1}$. We set $j_{t+2}=j$. Then $\left(1_{t+1}\right),\left(2_{t+1}\right),\left(3_{t+1}\right)$, and the minimality property hold for $\widetilde{Q}_{i}^{(t+1)}(x)$. In Section 5 we will slightly modify the construction of the polynomials $Q_{i}^{(0)}(x), \ldots, Q_{i}^{(n-1)}(x)$ (see (5.7) and Remark 5.8). Therefore we use the inequality $\left|a_{j_{l+1}}^{(l)}\right|>\xi^{n-1}\left|\widetilde{Q}_{i}^{(l)}\right|$ instead of $\left|a_{j_{l+1}}^{(l)}\right|=\widetilde{\widetilde{Q}_{i}^{(l)}},, 0 \leq l \leq n-1$.

In this way $\left(\widetilde{Q}_{i}^{(0)}(x), j_{1}\right), \ldots,\left(\widetilde{Q}_{i}^{(n-1)}(x), j_{n}\right)$ can be constructed. Clearly

$$
\left|\widetilde{Q}_{i}^{(0)}\right| \leq \widetilde{\widetilde{Q}_{i}^{(1)}} \leq \ldots \leq \widetilde{Q}_{i}^{(n-1)} .
$$

5. Properties of $\widetilde{Q}_{i}^{(0)}(x), \ldots, \widetilde{Q}_{i}^{(n-1)}(x)$. Using Lemma 3.1, the last two inequalities from (4.2), and (4.3), we deduce

$$
\widetilde{Q}_{i}^{(l)} \mid \leq c_{p}^{(l+1) /(n-l)}\left(\left|\widetilde{P}_{i-1}(\xi)\right| \prod_{\nu=0}^{l-1} \widetilde{\widetilde{Q}_{i}^{(\nu)} \mid}\right)^{-1 /(n-l)} \quad(1 \leq l \leq n-1) .
$$

Applying (4.3) to (5.1) with $l=n-1$, we get

$$
\left|\widetilde{Q}_{i}^{(n-1)}\right| \leq c_{p}^{n}\left|\widetilde{P}_{i-1}(\xi)\right|^{-1}\left(\prod_{\nu=0}^{n-2}\left|\widetilde{Q}_{i}^{(\nu)}\right|\right)^{-1} \leq c_{p}^{n}\left|\widetilde{P}_{i-1}(\xi)\right|^{-1}\left|\widetilde{P}_{i}\right|^{-n+1} .
$$

Similarly, (4.3) and (5.1) imply that

$$
\begin{aligned}
\left|\widetilde{Q}_{i}^{(l)}\right| & \leq\left|\widetilde{Q}_{i}^{(n-2)}\right| \leq c_{p}^{(n-1) / 2}\left|\widetilde{P}_{i-1}(\xi)\right|^{-1 / 2}\left(\prod_{\nu=0}^{n-3}\left|\widetilde{Q}_{i}^{(\nu)}\right|\right)^{-1 / 2} \\
& \leq c_{p}^{(n-1) / 2}\left|\widetilde{P}_{i-1}(\xi)\right|^{-1 / 2}\left|\widetilde{P}_{i}\right|^{1-n / 2} \quad(0 \leq l \leq n-2) .
\end{aligned}
$$


Lemma 5.1. Let $i$ be any natural number $>1$. Suppose that for some $0 \leq l \leq n-1$ the polynomial $\widetilde{Q}_{i}^{(l)}(x)$ satisfies the conditions of Lemma 3.2. Then

$$
\left|\widetilde{Q}_{i}^{(l)}\right|^{-1}<\left(c_{T} c_{p} \xi^{n-1}\right)^{-1 /(A-1)}\left|\widetilde{P}_{i-1}(\xi)\right|^{1 /(A-1)} .
$$

Proof. By Lemma 3.2 we obtain $\left|\widetilde{Q}_{i}^{(l)}\right|<\xi^{-n+1}\left|\widetilde{Q}_{i}^{(l) \prime}(\xi)\right|$. On the other hand, $\left|\widetilde{Q}_{i}^{(l)}\right|>\widetilde{H}_{0}$. Therefore by $(2.3)$ and $\left(1_{l}\right)$ we get

$$
c_{T}\left|\widetilde{Q}_{i}^{(l)}\right|^{-A}<\frac{\left|\widetilde{Q}_{i}^{(l)}(\xi)\right|}{\left|\widetilde{Q}_{i}^{(l) \prime}(\xi)\right|}<\xi^{-n+1} \frac{\left|\widetilde{Q}_{i}^{(l)}(\xi)\right|}{\left|\widetilde{Q}_{i}^{(l)}\right|}<c_{p}^{-1} \xi^{-n+1}\left|\widetilde{P}_{i-1}(\xi)\right|{\widetilde{Q_{i}^{(l)}}}^{-1}
$$

hence

$$
\left.{\widetilde{Q_{i}}}_{i}^{(l)}\right|^{-A+1}<c_{T}^{-1} c_{p}^{-1} \xi^{-n+1}\left|\widetilde{P}_{i-1}(\xi)\right|,
$$

and the result follows.

Define

$$
\begin{aligned}
c_{M}= & \min _{\substack{P(x) \in \mathbb{Z}[x], P(x) \not \equiv 0 \\
\operatorname{deg} P(x) \leq n,|P| \leq e^{n}\left|\widetilde{P}_{1}\right|}}(|P(\xi)|), \\
H_{0}= & c_{M}^{-30 n} c_{R}^{15 n} e^{60 n^{2}}\left|\widetilde{P}_{1}\right|^{n} .
\end{aligned}
$$

By (4.1)(ii) there exists an index $k_{0} \in \mathbb{N}$ such that $\left|\widetilde{P}_{k_{0}} \leq H_{0}<\right| \widetilde{P}_{k_{0}+1}$. From now on

$$
Q_{i}^{(l)}(x)=\widetilde{Q}_{k_{0}+i}^{(l)}(x) \quad \text { for any } i \in \mathbb{N} \text { and } l=0, \ldots, n-1 .
$$

In particular,

$$
P_{i}(x)=\widetilde{P}_{k_{0}+i}(x) \quad \text { for any } i \in \mathbb{N} .
$$

Lemma 5.2. For any natural $i>1$ we have

$$
\begin{aligned}
& \text { (I) }\left|\widetilde{P}_{i-1}(\xi)\right|<\left|\widetilde{P}_{i}\right|^{-(n-1)(A-1) /(A-2)}, \\
& \text { (II) } \prod_{\nu=0}^{n-2}\left|Q_{i}^{(\nu)}\right|<c_{p}^{-n}\left|P_{i-1}(\xi)\right|^{-(A-2) /(A-1)} .
\end{aligned}
$$

Pr o of. It follows from $\left(22_{l}\right)$ with $l=n-1$ and (4.3) that the polynomials $\widetilde{Q}_{i}^{(n-1)}(x)$ satisfy the conditions of Lemma 3.2. Substituting (5.2) into (5.4), we get

$$
\left(c_{p}^{n}\left|\widetilde{P}_{i-1}(\xi)\right|^{-1}\left|\widetilde{P}_{i}\right|^{-n+1}\right)^{-1}<\left(c_{T} c_{p} \xi^{n-1}\right)^{-1 /(A-1)}\left|\widetilde{P}_{i-1}(\xi)\right|^{1 /(A-1)},
$$

hence

$$
\left|\widetilde{P}_{i-1}(\xi)\right|^{(A-2) /(A-1)}<c_{p}^{n}\left(c_{T} c_{p} \xi^{n-1}\right)^{-1 /(A-1)}\left|\widetilde{P}_{i}\right|^{-n+1}
$$


and so, by the definitions of $c_{T}$ and $c_{p}$, we obtain

$$
\left|\widetilde{P}_{i-1}(\xi)\right|^{(A-2) /(A-1)}<\left.\widetilde{P}_{i}\right|^{-n+1},
$$

which gives (5.8)(I).

Similarly, substituting (5.1) with $l=n-1$ into (5.4) and keeping (5.7) in mind, we deduce

$$
\left(c_{p}^{n}\left|P_{i-1}(\xi)\right|^{-1}\left(\prod_{\nu=0}^{n-2}\left|Q_{i}^{(\nu)}\right|\right)^{-1}\right)^{-1}<\left(c_{T} c_{p} \xi^{n-1}\right)^{-1 /(A-1)}\left|P_{i-1}(\xi)\right|^{1 /(A-1)},
$$

hence

$$
\prod_{\nu=0}^{n-2}\left|\overline{Q_{i}^{(\nu)}}\right|<c_{p}^{n}\left(c_{T} c_{p} \xi^{n-1}\right)^{-1 /(A-1)}\left|P_{i-1}(\xi)\right|^{-(A-2) /(A-1)} .
$$

Using the definitions of $c_{T}$ and $c_{p}$, we get (5.8)(II).

Corollary 5.3. For any natural $i>1$ we have

$$
\begin{aligned}
& \text { (I) }\left|P_{i-1}(\xi)\right|<\left|P_{i}\right|^{-(n-1)(A-1) /(A-2)}, \\
& \text { (II) }\left|P_{i-1}(\xi)\right|<\left|P_{i}\right|^{-n} .
\end{aligned}
$$

Proof. The inequality (5.9)(I) immediately follows from (5.7) and (5.8)(I). To obtain (5.9)(II) we must use (5.9)(I) and the inequality $A<$ $n+1$ :

$$
\left|P_{i-1}(\xi)\right|<\left|P_{i}\right|^{-(n-1)(A-1) /(A-2)}<\left.\left.\right|_{P_{i}}\right|^{-(n-1)(n+1-1) /(n+1-2)}=\left|P_{i}\right|^{-n} .
$$

Lemma 5.4. For any $i \in \mathbb{N}$ the polynomials $P_{i}(x)$ are irreducible over $\mathbb{Z}$ and have degree $n$.

Proof. Assume that $P_{i}(x)=P_{i_{1}}(x) \ldots P_{i_{\gamma}}(x), 1 \leq \gamma \leq n$, where $P_{i_{1}}(x), \ldots, P_{i_{\gamma}}(x)$ are irreducible over $\mathbb{Z}$, have degree $<n$ and integer coefficients. Let the heights of $P_{i_{1}}(x), \ldots, P_{i_{\lambda}}(x)$ be greater than $e^{n}\left|\widetilde{P}_{1}\right|$ and the heights of the others be at most $e^{n} \widetilde{P}_{1}$. It is obvious that $\lambda \leq n$. We now show that $\lambda \geq 1$. In fact, assume that the heights of $P_{i_{1}}(x), \ldots, P_{i_{\gamma}}(x)$ do not exceed $e^{n}\left|\widetilde{P}_{1}\right|$. Then by (3.5) we get

$$
\left|P_{i}\right| \leq(n+1)^{n-1}\left|\widetilde{P_{i_{1}}} \ldots\right| \overrightarrow{P_{i_{\gamma}}} \mid \leq(n+1)^{n-1}\left(e^{n}\left|\widetilde{P_{1}}\right|\right)^{n},
$$

hence $\left|P_{i}\right| \leq(n+1)^{n-1} e^{n^{2}}\left|\widetilde{P}_{1}\right|^{n}$. On the other hand, (5.6) and (5.7) yield

$$
\left|P_{i}\right|>c_{M}^{-30 n} c_{R}^{15 n} e^{60 n^{2}}\left|\widetilde{P}_{1}\right|^{n}
$$

for any $i \in \mathbb{N}$. This gives a contradiction. 
We now prove that there exists an index $1 \leq j_{0} \leq \lambda$ such that

$$
\left|P_{i_{j_{0}}}(\xi)\right|<c_{R}^{-1 / 2} \mid P_{i_{j_{0}}}{ }^{-(n-1)(A-1) /(A-2)+1 / 30} .
$$

Assume the contrary. Then by (5.9)(I), the definition of $c_{M},(3.5)$, and (5.10) we have

$$
\begin{aligned}
& \left|P_{i+1}\right|^{-(n-1)(A-1) /(A-2)} \\
& \quad>\left|P_{i}(\xi)\right|=\prod_{\nu=1}^{\gamma}\left|P_{i_{\nu}}(\xi)\right| \geq c_{M}^{\gamma-\lambda} \prod_{\nu=1}^{\lambda}\left|P_{i_{\nu}}(\xi)\right| \\
& \quad \geq c_{M}^{\gamma-\lambda} c_{R}^{-\lambda / 2}\left(\prod_{\nu=1}^{\lambda}\left|P_{i_{\nu}}\right|\right)^{-(n-1)(A-1) /(A-2)+1 / 30} \\
& \quad>c_{M}^{n} c_{R}^{-n / 2}\left(e^{n}\left|P_{i}\right|\right)^{-(n-1)(A-1) /(A-2)+1 / 30} \\
& \quad=c_{M}^{n} c_{R}^{-n / 2} e^{-n(n-1)(A-1) /(A-2)+n / 30}\left|P_{i}\right|^{1 / 30}\left|P_{i}\right|^{-(n-1)(A-1) /(A-2)} \\
& >\left|P_{i}\right|^{-(n-1)(A-1) /(A-2)},
\end{aligned}
$$

which is impossible.

Since $1 \leq j_{0} \leq \lambda$, we have $\overline{P_{i_{0}}}>e^{n} \mid \widetilde{P}_{1}$. Therefore there exists an index $k \in \mathbb{N}$ such that

$$
e^{n}\left|\widetilde{P}_{k}\right|<\longdiv { P _ { i _ { 0 } } } \leq e ^ { n } | \widetilde { P } _ { k + 1 } | .
$$

Combining (5.8)(I) with (5.12), then using the inequality $\mid{\overrightarrow{P_{j_{0}}}}_{1}>\widetilde{H}_{0}>$ $c_{R}^{15} e^{60 n^{2}}$, we obtain

$$
\begin{aligned}
\left|\widetilde{P}_{k}(\xi)\right| & <{\widetilde{\widetilde{P}_{k+1}}}^{-(n-1)(A-1) /(A-2)} \leq\left(e^{-n} \mid \overline{P_{i_{0}}}\right)^{-(n-1)(A-1) /(A-2)} \\
& =e^{n(n-1)(A-1) /(A-2)}{\sqrt{P_{i_{0}}}}^{-1 / 30}{\sqrt{P_{i_{0}}}}^{-(n-1)(A-1) /(A-2)+1 / 30} \\
& <c_{R}^{-1 / 2}{\overline{P_{i_{0}}}}^{-(n-1)(A-1) /(A-2)+1 / 30} .
\end{aligned}
$$

Since $\sqrt{P_{i_{j_{0}}}}>e^{n} \mid \widetilde{P}_{k}$ and $P_{i_{j_{0}}}(x)$ is irreducible over $\mathbb{Z}$, by Lemma 3.6 the polynomials $\widetilde{P}_{k}(x)$ and $P_{i_{j_{0}}}(x)$ have no common root. Moreover, $\operatorname{deg} \widetilde{P}_{k}(x) \geq$ 2 and $\operatorname{deg} P_{i_{j_{0}}}(x) \geq 2$, since otherwise we get

$$
\frac{\left|\widetilde{P}_{k}(\xi)\right|}{\left|\widetilde{P}_{k}^{\prime}(\xi)\right|}=\frac{\left|\widetilde{P}_{k}(\xi)\right|}{\left|\widetilde{P}_{k}\right|}<\left|\widetilde{P}_{k}\right|^{-(n-1)(A-1) /(A-2)+1 / 30-1},
$$

and a simple calculation shows that

$$
-(n-1) \frac{A-1}{A-2}-\frac{29}{30}<-A,
$$


hence

$$
\frac{\left|\widetilde{P}_{k}(\xi)\right|}{\left|\widetilde{P}_{k}^{\prime}(\xi)\right|}<{\widetilde{P_{k}}}^{-A}
$$

which contradicts $(2.3)$. The same holds for $P_{i_{0}}(x)$. Thus, we can apply (3.2) to $\widetilde{P}_{k}(x)$ and $P_{i_{j}}(x)$.

(a) Substituting (5.11) and (5.13) into (3.2)(I), then using (5.12), we deduce

$$
\begin{aligned}
1 & <c_{R} \max \left(\left|\widetilde{P}_{k}(\xi)\right|,\left|P_{i_{j_{0}}}(\xi)\right|\right)^{2} \max \left(\left|\widetilde{P}_{k}\right|, \mid P_{i_{j_{0}}}\right)^{2 n-3} \\
& <\left.c_{R} c_{R}^{-1}\left|P_{i_{j_{0}}}-2(n-1)(A-1) /(A-2)+1 / 15\right|_{P_{i_{0}}}\right|^{2 n-3} \\
& ={ }_{P_{i_{0}}}{ }^{-2(n-1)(A-1) /(A-2)+2 n-44 / 15} .
\end{aligned}
$$

Here we have used the inequalities $\operatorname{deg} \widetilde{P}_{k}(x) \leq n, \operatorname{deg} P_{i_{j_{0}}}(x) \leq n-1$. It is easy to verify that

$$
-2(n-1) \frac{A-1}{A-2}+2 n-\frac{44}{15}<0 \quad \text { for } n=3, \ldots, 7,
$$

and we obtain a contradiction.

Since $\min \left(\left|\widetilde{P}_{k}\right|,\left|P_{i_{j_{0}}}\right|\right)>\widetilde{H}_{0}$, we can apply $(2.3)$ to the polynomials $\widetilde{P}_{k}(x)$ and $P_{i_{j_{0}}}(x)$.

(b) Applying (2.3) to (3.2)(II)-(III), then using (5.11)-(5.13) and the definitions of $c_{T}$ and $c_{R}$, we have

$$
\begin{aligned}
1 & <c_{R} c_{T}^{-2} \max \left(\left|\widetilde{P}_{k}(\xi)\right|,\left|P_{i_{j_{0}}}(\xi)\right|\right)^{3} \max \left(\left.\left|\widetilde{P}_{k},\right|\right|_{i_{j_{0}}}\right)^{2 A} \max \left(\left|\widetilde{P}_{k}\right|, \mid P_{i_{j_{0}}}\right)^{2 n-4} \\
& <\left.c_{R} c_{R}^{-3 / 2} c_{T}^{-2}\right|_{P_{i_{0}}}{ }^{-3(n-1)(A-1) /(A-2)+1 / 10}{\mid P_{i_{j_{0}}}}^{2 A+2 n-4} \\
& <P_{i_{j_{0}}}-3(n-1)(A-1) /(A-2)+2 A+2 n-39 / 10 .
\end{aligned}
$$

Since

$$
-3(n-1) \frac{A-1}{A-2}+2 A+2 n-\frac{39}{10}<0 \quad \text { for } n=3, \ldots, 7,
$$

we come to a contradiction again. This completes the proof.

Lemma 5.5. For any natural $i>1$ we have

$$
\left|P_{i-1}(\xi)\right|^{-1}<\left.\right|_{P_{i}}{ }^{(2 A+n-2) / 3}{\left|P_{i-1}\right|^{(n-1) / 3} .} .
$$

Pr o o f. By Lemma 5.4 the polynomials $P_{i-1}(x)$ and $P_{i}(x)$ are irreducible over $\mathbb{Z}$ and have degree $n$. Therefore they have no common root. Moreover, $\operatorname{deg} P_{i-1}(x) \geq 2$ and $\operatorname{deg} P_{i}(x) \geq 2$, since otherwise by (5.9)(II) we get

$$
\frac{\left|P_{i}(\xi)\right|}{\left|P_{i}^{\prime}(\xi)\right|}=\frac{\left|P_{i}(\xi)\right|}{\left|P_{i}\right|}<\left|P_{i}\right|^{-n-1},
$$


which contradicts (2.3). The same holds for $P_{i-1}(x)$. Thus, we can apply (3.2) to $P_{i-1}(x)$ and $P_{i}(x)$.

(a) Substituting (5.9)(II) into (3.2)(I) and using (4.1)(ii), we obtain

$$
\begin{aligned}
1 & <c_{R} \max \left(\left|P_{i-1}(\xi)\right|,\left|P_{i}(\xi)\right|\right)^{2} \max \left(\left|P_{i-1}\right|,\left|P_{i}\right|\right)^{2 n-2} \\
& <c_{R}\left|P_{i}\right|^{-2 n}\left|P_{i}\right|^{2 n-2}=c_{R}\left|P_{i}\right|^{-2},
\end{aligned}
$$

hence $\left|P_{i}\right|^{2}<c_{R}$. This gives a contradiction with (5.10).

Since $\min \left(\left|P_{i-1},\right| P_{i} \mid\right)>\widetilde{H}_{0}$, we can apply $(2.3)$ to the polynomials $P_{i-1}(x)$ and $P_{i}(x)$.

(b) Applying (2.3) to (3.2)(II), then using (4.1)(i), (4.1)(ii), and the definitions of $c_{T}$ and $c_{R}$, we deduce

$$
\begin{aligned}
1 & <c_{R} \max \left(\left|P_{i-1}(\xi)\right| \cdot\left|P_{i-1}^{\prime}(\xi)\right| \cdot\left|P_{i}^{\prime}(\xi)\right|,\left|P_{i}(\xi)\right| \cdot\left|P_{i-1}^{\prime}(\xi)\right|^{2}\right)\left|P_{i-1}\right|^{n-2}\left|P_{i}\right|^{n-1} \\
& <\left.\left.\left.\left.c_{R} c_{T}^{-2}\left|P_{i-1}(\xi)\right|^{3}\right|_{i-1}{ }^{A}\right|_{P_{i}}\right|^{A}\right|_{P_{i-1}} ^{n-2}\left|P_{i}\right|^{n-1} \\
& =c_{R} c_{T}^{-2}\left|P_{i-1}(\xi)\right|^{3}\left|P_{i}\right|^{A+n-1}\left|P_{i-1}\right|^{A+n-2} \\
& <\left|P_{i-1}(\xi)\right|^{3}\left|P_{i}\right|^{2 A+n-2}\left|P_{i-1}\right|^{n-1} .
\end{aligned}
$$

(c) Similarly, by (2.3), (3.2)(III), (4.1)(i), (4.1)(ii), and the definitions of $c_{T}$ and $c_{R}$, we have

$$
\begin{aligned}
& 1<c_{R} \max \left(\left|P_{i}(\xi)\right| \cdot\left|P_{i-1}^{\prime}(\xi)\right| \cdot\left|P_{i}^{\prime}(\xi)\right|,\left|P_{i-1}(\xi)\right| \cdot\left|P_{i}^{\prime}(\xi)\right|^{2}\right)\left|P_{i-1}\right|^{n-1}\left|P_{i}\right|^{n-2}
\end{aligned}
$$

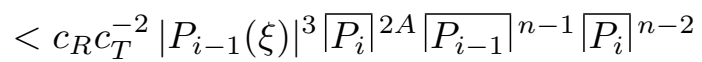

$$
\begin{aligned}
& =c_{R} c_{T}^{-2}\left|P_{i-1}(\xi)\right|^{3}{\mid P_{i}}^{2 A+n-2}\left|P_{i-1}\right|^{n-1}
\end{aligned}
$$

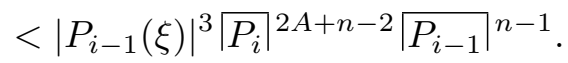

It is easy to see that either one of the above two inequalities gives (5.14).

Lemma 5.6. For any natural $i>1$ we have

$$
\prod_{\nu=0}^{n-2}\left|Q_{i}^{(\nu)}\right|<c_{p}^{-n}\left|P_{i-1}(\xi)\right|^{-1 / 2}\left|P_{i}\right|^{-1+n / 2} .
$$

Pr o o f. From (5.8)(II) we deduce

$$
\begin{aligned}
& \prod_{\nu=0}^{n-2}\left|Q_{i}^{(\nu)}\right| \\
& <c_{p}^{-n}\left|P_{i-1}(\xi)\right|^{-(A-2) /(A-1)} \\
& \equiv c_{p}^{-n}\left|P_{i-1}(\xi)\right|^{-1 / 2}\left|P_{i}\right|^{-1+n / 2}\left|P_{i-1}(\xi)\right|^{-(A-3) /(2(A-1))}\left|P_{i}\right|^{1-n / 2} .
\end{aligned}
$$

We now prove that

$$
\left|P_{i-1}(\xi)\right|^{-(A-3) /(2(A-1))}\left|P_{i}\right|^{1-n / 2}<1 .
$$


If the result were false, we should have

$$
\left|P_{i-1}(\xi)\right| \leq\left|P_{i}\right|^{-(n-2)(A-1) /(A-3)} .
$$

Substituting this into (5.14), we get

$$
\begin{aligned}
1 & <\left.\left.\left|P_{i-1}(\xi)\right|\right|_{i}\right|^{(2 A+n-2) / 3}\left|\vec{P}_{i-1}\right|^{(n-1) / 3} \\
& \leq\left|P_{i}\right|^{-(n-2)(A-1) /(A-3)+(2 A+n-2) / 3}\left|P_{i-1}\right|^{(n-1) / 3} \\
& <\left|P_{i}\right|^{-(n-2)(A-1) /(A-3)+(2 A+n-2) / 3+(n-1) / 3} .
\end{aligned}
$$

A simple calculation shows that

$$
-\frac{(n-2)(A-1)}{A-3}+\frac{2 A+2 n-3}{3}<0 \quad \text { for } n=3, \ldots, 7,
$$

and we obtain a contradiction. This gives (5.17). Finally, (5.16) and (5.17) imply (5.15).

LEMmA 5.7. Let $i$ be any natural number $>1$. Then for any $0 \leq l \leq n-2$ there exist at least two indices $\left\{k_{1}, k_{2}\right\} \subset\{1, \ldots, n\}$ such that

$$
\left|a_{k_{\nu}}^{(l)}\right|>\xi^{n-1}\left|Q_{i}^{(l)}\right| \quad(\nu=1,2) .
$$

Proof. By Lemma 3.1 for any $0 \leq l \leq n-1$ there exists an index $k_{1} \in\{1, \ldots, n\}$ such that $\left|a_{k_{1}}^{(l)}\right|=\left|Q_{i}^{(l)}\right|$.

Fix some $0 \leq l \leq n-2$ and suppose that $\left|a_{k}^{(l)}\right| \leq \xi^{n-1}\left|Q_{i}^{(l)}\right|$ for all $k \in\{1, \ldots, n\} \backslash\left\{k_{1}\right\}$. Then the polynomial $Q_{i}^{(l)}(x)$ satisfies the conditions of Lemma 3.2. Therefore we can apply Lemma 5.1 to $Q_{i}^{(l)}(x)$. Substituting (5.3) into (5.4) and keeping (5.7) in mind, we obtain

$$
\left(c_{p}^{(n-1) / 2}\left|P_{i-1}(\xi)\right|^{-1 / 2}\left|P_{i}\right|^{1-n / 2}\right)^{-1}<\left(c_{T} c_{p} \xi^{n-1}\right)^{-1 /(A-1)}\left|P_{i-1}(\xi)\right|^{1 /(A-1)} .
$$

This inequality can be written as

$$
\left|P_{i-1}(\xi)\right|^{(A-3) /(2(A-1))}\left|P_{i}\right|^{-1+n / 2}<\left(c_{T} c_{p} \xi^{n-1}\right)^{-1 /(A-1)} c_{p}^{(n-1) / 2},
$$

and so, by the definitions of $c_{T}$ and $c_{p}$, we get

$$
\left|P_{i-1}(\xi)\right|^{(A-3) /(2(A-1))}\left|P_{i}\right|^{-1+n / 2}<1,
$$

which contradicts (5.17).

REMARK 5.8. We now can slightly modify the construction of the polynomials $Q_{i}^{(0)}(x), \ldots, Q_{i}^{(n-1)}(x)$. By Lemma 5.7 there are at least two indices $\left\{k_{1}, k_{2}\right\} \subset\{1, \ldots, n\}$ such that

$$
\left|a_{k_{\nu}}^{(0)}\right|>\xi^{n-1} \mid \widehat{Q_{i}^{(0)} \mid} \quad(\nu=1,2) .
$$

We may suppose that $k_{1} \in\{1, \ldots, n-1\}$ and set $j_{1}=k_{1}$. We now construct $\left(Q_{i}^{(1)}(x), j_{2}\right), \ldots,\left(Q_{i}^{(n-1)}(x), j_{n}\right)$ with this (possibly new) value of $j_{1}$. Again 
there are at least two indices $\left\{k_{1}, k_{2}\right\} \subset\{1, \ldots, n\}$ with

$$
\left|a_{k_{\nu}}^{(1)}\right|>\xi^{n-1} \mid \widehat{Q_{i}^{(1)}} \quad(\nu=1,2) .
$$

Since $\left|a_{j_{1}}^{(1)}\right| \leq c_{p}^{-1} \widehat{Q_{i}^{(0)} \mid}<\xi^{n-1}\left|Q_{i}^{(1)}\right|$, these indices are distinct from $j_{1}$. So, we can pick $j_{2} \in\{1, \ldots, n-1\} \backslash\left\{j_{1}\right\}$, etc. In this way we can arrange $j_{1}, \ldots, j_{n-1}$ so that $\left\{j_{1}, \ldots, j_{n-1}\right\}=\{1, \ldots, n-1\}$. Below, we assume this is true.

6. Three statements. The following results are of great importance for this paper.

StATEMEnT 6.1. Let $i$ be any natural number $>1$. Write

$$
P_{i-1}(x)=b_{n} x^{n}+\ldots+b_{1} x+b_{0} .
$$

Then the polynomials $P_{i-1}(x), Q_{i}^{(0)}(x), \ldots, Q_{i}^{(n-2)}(x)$ are linearly independent and also

$$
|\Delta|=\left\|\begin{array}{cccc}
a_{j_{1}}^{(n-2)} & \ldots & a_{j_{n-1}}^{(n-2)} & Q_{i}^{(n-2)}(\xi) \\
\ldots \ldots \ldots \ldots & \ldots & \ldots \ldots \ldots & \ldots \ldots \\
a_{j_{1}}^{(0)} & \ldots & a_{j_{n-1}}^{(0)} & Q_{i}^{(0)}(\xi) \\
b_{j_{1}} & \ldots & b_{j_{n-1}} & P_{i-1}(\xi)
\end{array}\right\|>\xi^{n^{2}}\left|P_{i-1}(\xi)\right| \prod_{\nu=0}^{n-2}\left|Q_{i}^{(\nu)}\right|
$$

where $j_{1}, \ldots, j_{n-1}$ are the indices of the $Q_{i}$-system.

Proof. From this moment on, we will take into account the notation (5.7) when using the formulas from Section 4 . By $\left(2_{l}\right)$ with $1 \leq l \leq n-2$ and (4.3) we have

$$
\left|a_{j_{\mu}}^{(l)}\right| \leq c_{p}^{-1}\left|Q_{i}^{(\mu-1)}\right| \leq c_{p}^{-1}\left|Q_{i}^{(l)}\right| \quad(1 \leq \mu \leq l),
$$

hence

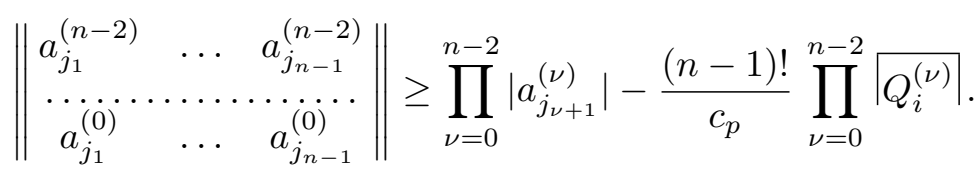

Applying $\left(3_{l}\right)$ with $l=0, \ldots, n-2$ to $\prod_{\nu=0}^{n-2}\left|a_{j_{\nu+1}}^{(\nu)}\right|$, we obtain

$$
\begin{aligned}
\left\|\begin{array}{ccc}
a_{j_{1}}^{(n-2)} & \ldots & a_{j_{n-1}}^{(n-2)} \\
\ldots \ldots \ldots \ldots & \ldots \ldots & \ldots \\
a_{j_{1}}^{(0)} & \ldots & a_{j_{n-1}}^{(0)}
\end{array}\right\| & \geq \xi^{(n-1)^{2}} \prod_{\nu=0}^{n-2}\left|Q_{i}^{(\nu)}\right|-\frac{(n-1) !}{c_{p}} \prod_{\nu=0}^{n-2} Q_{i}^{(\nu)} \mid \\
& =\left(\xi^{(n-1)^{2}}-\frac{(n-1) !}{c_{p}}\right) \prod_{\nu=0}^{n-2} \mid Q_{i}^{(\nu)} .
\end{aligned}
$$

On the other hand, by (4.1)(ii) and (4.3) the absolute values of other minors from the first $n-1$ columns of the determinant $\Delta$ are less than or 
equal to $(n-1) ! \prod_{\nu=0}^{n-2}\left|Q_{i}^{(\nu)}\right|$. Hence by $\left(1_{l}\right)$ with $l=0, \ldots, n-2,(6.2)$ and the definition of $c_{p}$, we get

$$
\begin{aligned}
|\Delta|> & \left(\xi^{(n-1)^{2}}-\frac{(n-1) !}{c_{p}}\right)\left|P_{i-1}(\xi)\right| \prod_{\nu=0}^{n-2}\left|Q_{i}^{(\nu)}\right| \\
& -(n-1) !\left(\sum_{\nu=0}^{n-2}\left|Q_{i}^{(\nu)}(\xi)\right|\right) \prod_{\nu=0}^{n-2}\left|Q_{i}^{(\nu)}\right| \\
> & \left(\xi^{(n-1)^{2}}-\frac{(n-1) !}{c_{p}}\right)\left|P_{i-1}(\xi)\right| \prod_{\nu=0}^{n-2}\left|Q_{i}^{(\nu)}\right| \\
& -\frac{(n-1) !(n-1)}{c_{p}}\left|P_{i-1}(\xi)\right| \prod_{\nu=0}^{n-2}\left|Q_{i}^{(\nu)}\right| \\
> & \xi^{n^{2}}\left|P_{i-1}(\xi)\right| \prod_{\nu=0}^{n-2} Q_{i}^{(\nu)} .
\end{aligned}
$$

This gives (6.1). Finally, since $|\Delta|>0$, the polynomials $P_{i-1}(x), Q_{i}^{(0)}(x), \ldots$ $\ldots, Q_{i}^{(n-2)}(x)$ are linearly independent.

StATEMENT 6.2. Let $i$ and $\tau$ be natural numbers such that

$$
\left|P_{i-1} \leq c_{h}\right| P_{\tau}, \quad 1 \leq \tau \leq i-1, i>1,
$$

where

$$
c_{h}=4(n !)^{2} c_{p}^{2 n}
$$

Let also $L(x)$ be a nonzero polynomial satisfying

$$
\begin{aligned}
|L(\xi)| & <\left|P_{i-1}(\xi)\right|^{1 / 2}\left|P_{i}\right|^{-1+n / 2}\left|P_{\tau}\right|^{-n+1}, \\
\left|L^{\prime}(\xi)\right| & <\left|P_{i-1}(\xi)\right|^{1-A / 2}\left|P_{i}\right|^{(n-2)(1-A / 2)}\left|P_{\tau}\right|^{-n+2}, \\
|L| & <\xi^{-n+1}\left|L^{\prime}(\xi)\right| .
\end{aligned}
$$

Then

$$
\frac{|L(\xi)|}{\left|L^{\prime}(\xi)\right|}<\left(c_{h} \xi^{-1}\right)^{(n-1) A}|L|^{-A}
$$

Proof. By (6.4), (6.3), (5.9)(II), and (5.14) we get

$$
\begin{aligned}
|L(\xi)|< & \left|P_{i-1}(\xi)\right|^{1 / 2}\left|P_{i}\right|^{-1+n / 2}\left|P_{\tau}\right|^{-n+1} \\
\leq & c_{h}^{n-1}\left|P_{i-1}(\xi)\right|^{1 / 2}\left|P_{i}\right|^{-1+n / 2}\left|P_{i-1}\right|^{-n+1} \\
= & c_{h}^{n-1}\left|P_{i-1}(\xi)\right|^{1 / 2+\alpha_{1}-\alpha_{2}}\left|P_{i-1}(\xi)\right|^{-\alpha_{1}}\left|P_{i-1}(\xi)\right|^{\alpha_{2}} \\
& \times\left|P_{i}\right|^{-1+n / 2}\left|P_{i-1}\right|^{-n+1} \\
< & c_{h}^{n-1}\left|P_{i-1}(\xi)\right|^{1 / 2+\alpha_{1}-\alpha_{2}}\left|P_{i}\right|^{(2 A+n-2) \alpha_{1} / 3}\left|P_{i-1}\right|^{(n-1) \alpha_{1} / 3} \\
& \times\left|P_{i}\right|^{-n \alpha_{2}}\left|P_{i}\right|^{-1+n / 2}\left|P_{i-1}\right|^{-n+1}
\end{aligned}
$$




$$
\begin{aligned}
= & c_{h}^{n-1}\left|P_{i-1}(\xi)\right|^{1 / 2+\alpha_{1}-\alpha_{2}}\left|P_{i}\right|^{(2 A+n-2) \alpha_{1} / 3-n \alpha_{2}-1+n / 2} \\
& \times\left|P_{i-1}\right|^{(n-1) \alpha_{1} / 3-n+1},
\end{aligned}
$$

where $\alpha_{1}$ and $\alpha_{2}$ are any nonnegative constants. Put

$$
\begin{aligned}
& \alpha_{1}=\frac{3(n-2)(A-1)}{n-1}+3, \\
& \alpha_{2}=\frac{7}{2}+\frac{3(n-2)(A-1)}{n-1}-\left(\frac{A}{2}-1\right)(A-1) .
\end{aligned}
$$

It is easy to verify that for $n=3, \ldots, 7$ the constants $\alpha_{1}$ and $\alpha_{2}$ are positive. By (6.9) we have

$$
\frac{1}{2}+\alpha_{1}-\alpha_{2}=\left(\frac{A}{2}-1\right)(A-1), \quad \frac{n-1}{3} \alpha_{1}-n+1=(n-2)(A-1),
$$

and

$$
\begin{aligned}
& \frac{2 A+n-2}{3} \alpha_{1}-n \alpha_{2}-1+\frac{n}{2} \\
& =\frac{n^{2} A^{2}+3 n A^{2}-7 n^{2} A+7 n A-8 A^{2}+12 A+2 n^{2}-8 n-2}{2(n-1)} \\
& \equiv \frac{2\left((3 n-5) A^{2}-\left(2 n^{2}+n-9\right) A-n-3\right)+(n-1)(n-2)(A-2)(A-1)}{2(n-1)},
\end{aligned}
$$

hence by (1.3) we obtain

$$
\begin{aligned}
& \frac{2 A+n-2}{3} \alpha_{1}-n \alpha_{2}-1+\frac{n}{2} \\
& \quad=\frac{(n-1)(n-2)(A-2)(A-1)}{2(n-1)}=(n-2)\left(\frac{A}{2}-1\right)(A-1) .
\end{aligned}
$$

Finally, (6.8), (6.10), and (6.11) imply that

$$
\begin{aligned}
& |L(\xi)|<c_{h}^{n-1}\left|P_{i-1}(\xi)\right|^{(A / 2-1)(A-1)} \\
& \times\left.\right|_{P_{i}}{ }^{(n-2)(A / 2-1)(A-1)}\left|P_{i-1}\right|^{(n-2)(A-1)} .
\end{aligned}
$$

On the other hand, if we raise both sides of (6.5) to the power $-A+1$ and apply (6.3), we get

$$
\begin{aligned}
\left|L^{\prime}(\xi)\right|^{-A+1}> & \left|P_{i-1}(\xi)\right|^{(A / 2-1)(A-1)}\left|P_{i}\right|^{(n-2)(A / 2-1)(A-1)}\left|P_{\tau}\right|^{(n-2)(A-1)} \\
\geq & c_{h}^{-(n-2)(A-1)}\left|P_{i-1}(\xi)\right|^{(A / 2-1)(A-1)} \\
& \times\left|P_{i}\right|^{(n-2)(A / 2-1)(A-1)}\left|P_{i-1}\right|^{(n-2)(A-1)} .
\end{aligned}
$$

Combining this with (6.12), we find that $|L(\xi)|<c_{h}^{(n-1) A}\left|L^{\prime}(\xi)\right|^{-A+1}$. We now divide both sides of this inequality by $\left|L^{\prime}(\xi)\right|$ and apply (6.6): 


$$
\frac{|L(\xi)|}{\left|L^{\prime}(\xi)\right|}<c_{h}^{(n-1) A}\left|L^{\prime}(\xi)\right|^{-A}<c_{h}^{(n-1) A} \xi^{-(n-1) A}|L|^{-A},
$$

which gives (6.7).

Statement 6.3. Let $i$ and $\tau$ be as in Statement 6.2. Let also $A_{1}, \ldots, A_{n}$ be positive numbers such that

$$
\begin{gathered}
\prod_{\nu=1}^{n} A_{\nu} \geq n !\left|P_{i-1}(\xi)\right| \prod_{\nu=0}^{n-2}\left|Q_{i}^{(\nu)}\right|, \\
A_{1} \leq\left. c_{p}^{n}\left|P_{i-1}(\xi)\right| \prod_{\nu=0}^{n-2}\left|Q_{i}^{(\nu)}\right| P_{\tau}\right|^{-n+1},
\end{gathered}
$$

$$
\left|\widetilde{P}_{1}\right| \leq A_{2} \leq \ldots \leq A_{n} \leq c_{p}^{(n-3) / 2}\left|P_{i-1}(\xi)\right|^{-1 / 2}\left|P_{i}\right|^{-1+n / 2}\left|P_{\tau}\right|^{-n+2} .
$$

Then there exists a nonzero polynomial $L(x)=c_{n} x^{n}+\ldots+c_{1} x+c_{0}$ with integer coefficients which satisfies

$$
\begin{gathered}
|L(\xi)|<A_{1}, \\
\left|c_{k_{\nu}}\right| \leq A_{\nu+1} \quad(1 \leq \nu \leq n-1), \\
|L|<\xi^{-n+1} A_{n},
\end{gathered}
$$

where $\left\{k_{1}, \ldots, k_{n-1}\right\}=\{1, \ldots, n-1\}$.

Proof. First we note that by (5.9)(II), (6.3), and (4.1)(ii) we obtain

$$
\left|\widetilde{P}_{1}\right| \leq\left|P_{i-1}(\xi)\right|^{-1 / 2}\left|P_{i}\right|^{-1+n / 2}\left|P_{\tau}\right|^{-n+2},
$$

so $(6.15)$ is correct.

Consider the following system of inequalities:

$$
\left\{\begin{array}{l}
\left|\sum_{\nu=0}^{n-2} Q_{i}^{(\nu)}(\xi) x_{\nu}+P_{i-1}(\xi) x_{n-1}\right|<A_{1}, \\
\left|\sum_{\nu=0}^{n-2} a_{k_{1}}^{(\nu)} x_{\nu}+b_{k_{1}} x_{n-1}\right| \leq A_{2}, \\
\ldots \ldots \ldots \ldots \ldots \ldots \ldots \ldots \ldots \ldots \ldots \ldots \ldots \ldots \ldots \ldots \ldots \ldots \ldots \ldots \\
\left|\sum_{\nu=0}^{n-2} a_{k_{n-1}}^{(\nu)} x_{\nu}+b_{k_{n-1}} x_{n-1}\right| \leq A_{n}
\end{array}\right.
$$

We now prove that

$$
(6.20) \quad|\Delta|=\left\|\begin{array}{cccc}
Q_{i}^{(0)}(\xi) & \ldots & Q_{i}^{(n-2)}(\xi) & P_{i-1}(\xi) \\
a_{k_{1}}^{(0)} & \ldots & a_{k_{1}}^{(n-2)} & b_{k_{1}} \\
\ldots \ldots \ldots & \ldots \ldots & \ldots \ldots \ldots \ldots \ldots \ldots \\
a_{k_{n-1}}^{(0)} & \ldots & a_{k_{n-1}}^{(n-2)} & b_{k_{n-1}}
\end{array}\right\| \leq n !\left|P_{i-1}(\xi)\right| \prod_{\nu=0}^{n-2}\left|Q_{i}^{(\nu)}\right|
$$


In fact, it follows from $\left(1_{l}\right)$ with $l=0, \ldots, n-2$ that the entries of the first line of the determinant $\Delta$ are at most $\left|P_{i-1}(\xi)\right|$ in absolute value. On the other hand, (4.1)(ii) and (4.3) imply that any minor of the other $n-1$ lines has absolute value at most $(n-1) ! \prod_{\nu=0}^{n-2}\left|Q_{i}^{(\nu)}\right|$. This gives $(6.20)$.

Thanks to (6.13), (6.20), and Minkowski's Theorem on linear forms there exists a nonzero integer solution $\left(\widetilde{x}_{0}, \ldots, \widetilde{x}_{n-1}\right) \in \mathbb{Z}^{n}$ of (6.19). Using Remark 5.8 , we have $\left\{k_{1}, \ldots, k_{n-1}\right\}=\left\{j_{1}, \ldots, j_{n-1}\right\}$, where $j_{1}, \ldots, j_{n-1}$ are the indices of the $Q_{i}$-system. Therefore we can apply (6.1) to the determinant $\Delta$. It follows from $\left(1_{l}\right)$ with $l=0, \ldots, n-2,(4.1)($ ii), (4.3), (6.1), and (6.15) that the system (6.19) satisfies the conditions of Lemma 3.7. By this lemma and the definition of $c_{p}$ we have

$$
\begin{aligned}
\left|\widetilde{x}_{\nu}\right| & \leq c_{p} \max \left(\frac{A_{1}\left|P_{i-1}\right|}{\left|P_{i-1}(\xi)\right|\left|Q_{i}^{(\nu)}\right|}, \frac{A_{n}}{\left|Q_{i}^{(\nu)}\right|}\right) \quad(\nu=0, \ldots, n-2), \\
\left|\widetilde{x}_{n-1}\right| & \leq c_{p} \max \left(\frac{A_{1}}{\left|P_{i-1}(\xi)\right|}, \frac{A_{n}}{\left|P_{i-1}\right|}\right) .
\end{aligned}
$$

Put

$$
L(x)=\sum_{\nu=0}^{n-2} Q_{i}^{(\nu)}(x) \widetilde{x}_{\nu}+P_{i-1}(x) \widetilde{x}_{n-1}=c_{n} x^{n}+\ldots+c_{1} x+c_{0} .
$$

The polynomials $Q_{i}^{(0)}(x), \ldots, Q_{i}^{(n-2)}(x)$ and $P_{i-1}(x)$ have integer coefficients and by Statement 6.1 are linearly independent. On the other hand, the solution $\left(\widetilde{x}_{0}, \ldots, \widetilde{x}_{n-1}\right)$ is nonzero and integer. Hence the polynomial $L(x)$ is nonzero and has integer coefficients as well.

From (6.19) and (6.22) we deduce (6.16) and (6.17). Let us prove (6.18). We first obtain an upper bound for $|L(\xi)|$ and $\left|L^{\prime}(\xi)\right|$.

Applying (6.14) to (6.16) and using (5.15), we find that

$$
|L(\xi)|<\left|P_{i-1}(\xi)\right|^{1 / 2}\left|P_{i}\right|^{-1+n / 2}\left|P_{\tau}\right|^{-n+1} .
$$

Using (6.22), (2.3), (6.21), $\left(1_{l}\right)$ with $l=0, \ldots, n-2,(4.1)(\mathrm{ii})$, and (4.3), we get

$$
\begin{aligned}
\left|L^{\prime}(\xi)\right| \leq & \sum_{\nu=0}^{n-2}\left|Q_{i}^{(\nu) \prime}(\xi)\right| \cdot\left|\widetilde{x}_{\nu}\right|+\left|P_{i-1}^{\prime}(\xi)\right| \cdot\left|\widetilde{x}_{n-1}\right| \\
\leq & c_{T}^{-1} c_{p}\left(\sum_{\nu=0}^{n-2}\left|Q_{i}^{(\nu)}(\xi)\right| Q_{i}^{(\nu)} A \max \left(\frac{A_{1}\left|P_{i-1}\right|}{\left|P_{i-1}(\xi)\right| Q_{i}^{(\nu)} \mid}, \frac{A_{n}}{\left|Q_{i}^{(\nu)}\right|}\right)\right. \\
& \left.+\left|P_{i-1}(\xi)\right|\left|P_{i-1}\right|^{A} \max \left(\frac{A_{1}}{\left|P_{i-1}(\xi)\right|}, \frac{A_{n}}{\left|P_{i-1}\right|}\right)\right)
\end{aligned}
$$




$$
\begin{aligned}
& <c_{T}^{-1} c_{p}\left|P_{i-1}(\xi)\right|\left(\sum_{\nu=0}^{n-2}\left|Q_{i}^{(\nu)}\right| A-1+\left|P_{i-1}\right|^{A-1}\right) \\
& \quad \times \max \left(\frac{A_{1}\left|P_{i-1}\right|}{\left|P_{i-1}(\xi)\right|}, A_{n}\right) \\
& <n c_{T}^{-1} c_{p}\left|P_{i-1}(\xi)\right| Q_{i}^{(n-2)} \mid A-1 \max \left(\frac{A_{1}\left|P_{i-1}\right|}{\left|P_{i-1}(\xi)\right|}, A_{n}\right) .
\end{aligned}
$$

By (6.14), (5.15), and (6.3) we have

$$
\begin{aligned}
\frac{A_{1}\left|P_{i-1}\right|}{\left|P_{i-1}(\xi)\right|} & \leq c_{p}^{n} \prod_{\nu=0}^{n-2}\left|Q_{i}^{(\nu)}\right|\left|P_{\tau}\right|^{-n+1}\left|P_{i-1}\right| \\
& <\left|P_{i-1}(\xi)\right|^{-1 / 2}\left|P_{i}\right|^{-1+n / 2}\left|P_{\tau}\right|^{-n+1}\left|P_{i-1}\right| \\
& \leq c_{h}\left|P_{i-1}(\xi)\right|^{-1 / 2}\left|P_{i}\right|^{-1+n / 2}\left|P_{\tau}\right|^{-n+2}
\end{aligned}
$$

Substituting (6.15) and (6.25) into (6.24), then using (5.3) and the definitions of $c_{T}, c_{p}$ and $c_{h}$, we obtain

$$
\begin{aligned}
\left|L^{\prime}(\xi)\right|< & n c_{T}^{-1} c_{p} c_{h}\left|P_{i-1}(\xi)\right|^{1 / 2}\left|Q_{i}^{(n-2)}\right| A-1\left|P_{i}\right|^{-1+n / 2}\left|P_{\tau}\right|^{-n+2} \\
< & n c_{T}^{-1} c_{p} c_{h} c_{p}^{(n-1)(A-1) / 2}\left|P_{i-1}(\xi)\right|^{1 / 2}\left|P_{i-1}(\xi)\right|^{-(A-1) / 2} \\
& \times\left|P_{i}\right|^{(1-n / 2)(A-1)}\left|P_{i}\right|^{-1+n / 2}\left|P_{\tau}\right|^{-n+2} \\
< & \left|P_{i-1}(\xi)\right|^{1-A / 2}\left|P_{i}\right|^{(n-2)(1-A / 2)}\left|P_{\tau}\right|^{-n+2}
\end{aligned}
$$

Now we can complete the proof of (6.18). Assume that $|L| \geq \xi^{-n+1} A_{n}$. Hence by (6.15) and (6.17) we have $\left|c_{k_{\nu}}\right| \leq A_{\nu+1} \leq A_{n} \leq \xi^{n-1}|L|, \nu=$ $1, \ldots, n-1$. Therefore $L(x)$ satisfies the conditions of Lemma 3.2. Thus $|L|<\xi^{-n+1}\left|L^{\prime}(\xi)\right|$. Hence by (6.23) and (6.26) the polynomial $L(x)$ satisfies the conditions of Statement 6.2. It follows that

$$
\frac{|L(\xi)|}{\left|L^{\prime}(\xi)\right|}<\left(c_{h} \xi^{-1}\right)^{(n-1) A}|L|^{-A} .
$$

Since $|L| \geq \xi^{-n+1} A_{n}>\widetilde{P_{1}}$ and $c_{T}>\left(c_{h} \xi^{-1}\right)^{(n-1) A}$, we obtain a contradiction with (2.3). Hence $|L|<\xi^{-n+1} A_{n}$.

Corollary 6.4. For any natural $i>2$ we have

$$
\left|P_{\tau-1}(\xi)\right| \prod_{\nu=0}^{n-2}\left|Q_{\tau}^{(\nu)}\right|<n ! c_{p}^{n}\left|P_{i-1}(\xi)\right| \prod_{\nu=0}^{n-2}\left|Q_{i}^{(\nu)}\right|,
$$

where $P_{\tau-1}(x)$ is the polynomial from (4.1) with $\left|P_{i-1}\right| \leq c_{h}\left|P_{\tau}\right|, 1<\tau \leq$ $i-1$. 
Pr o of. Suppose that

$$
\left|P_{\tau-1}(\xi)\right| \prod_{\nu=0}^{n-2}\left|Q_{\tau}^{(\nu)}\right| \geq n ! c_{p}^{n}\left|P_{i-1}(\xi)\right| \prod_{\nu=0}^{n-2}\left|Q_{i}^{(\nu)}\right|
$$

for some natural $i>2$. Put

$$
\begin{aligned}
& A_{1}=\min \left(c_{p}^{-1}\left|P_{\tau-1}(\xi)\right|, c_{p}^{n}\left|P_{i-1}(\xi)\right| \prod_{\nu=0}^{n-2}\left|Q_{i}^{(\nu)}\right| \mid P_{\tau}{ }^{-n+1}\right), \\
& A_{\nu}=c_{p}^{-1}\left|Q_{\tau}^{(\nu-2)}\right| \quad(2 \leq \nu \leq n) .
\end{aligned}
$$

We now prove that $A_{1}, \ldots, A_{n}$ satisfy the conditions of Statement 6.3. In fact, if $A_{1}=c_{p}^{-1}\left|P_{\tau-1}(\xi)\right|$, then by (6.28)-(6.30) we get

$$
\begin{aligned}
\prod_{\nu=1}^{n} A_{\nu} & =c_{p}^{-n}\left|P_{\tau-1}(\xi)\right| \prod_{\nu=0}^{n-2}\left|Q_{\tau}^{(\nu)}\right| \geq c_{p}^{-n} n ! c_{p}^{n}\left|P_{i-1}(\xi)\right| \prod_{\nu=0}^{n-2}\left|Q_{i}^{(\nu)}\right| \\
& =n !\left|P_{i-1}(\xi)\right| \prod_{\nu=0}^{n-2} \mid Q_{i}^{(\nu)} .
\end{aligned}
$$

Similarly, if $A_{1}=c_{p}^{n}\left|P_{i-1}(\xi)\right| \prod_{\nu=0}^{n-2}\left|Q_{i}^{(\nu)}\right|\left|P_{\tau}\right|^{-n+1}$, then by (6.30), (4.3), and the definition of $c_{p}$,

$$
\prod_{\nu=1}^{n} A_{\nu}=\left.c_{p}\left|P_{i-1}(\xi)\right| \prod_{\nu=0}^{n-2}\left|Q_{i}^{(\nu)}\right| P_{\tau}\right|^{-n+1} \prod_{\nu=0}^{n-2}\left|Q_{\tau}^{(\nu)}\right|>n !\left|P_{i-1}(\xi)\right| \prod_{\nu=0}^{n-2}\left|Q_{i}^{(\nu)}\right| .
$$

By (6.29) we have

$$
A_{1} \leq c_{p}^{n}\left|P_{i-1}(\xi)\right| \prod_{\nu=0}^{n-2}\left|Q_{i}^{(\nu)}\right| \mid P_{\tau}{ }^{-n+1}
$$

Finally, by (6.30) and (5.3) we obtain

$$
\begin{aligned}
A_{n}= & \left.c_{p}^{-1}\left|\overline{Q_{\tau}^{(n-2)} \mid} \leq c_{p}^{(n-3) / 2}\right| P_{\tau-1}(\xi)\right|^{-1 / 2}\left|P_{\tau}\right|^{1-n / 2} \\
= & c_{p}^{(n-3) / 2}\left|P_{i-1}(\xi)\right|^{-1 / 2} \frac{\left|P_{\tau-1}(\xi)\right|^{-1 / 2}}{\left|P_{i-1}(\xi)\right|^{-1 / 2}} \\
& \times\left|P_{i}\right|^{-1+n / 2} \frac{\left|P_{\tau}\right|^{-1+n / 2}}{\left|P_{i}\right|^{-1+n / 2}}\left|P_{\tau}\right|^{-n+2} .
\end{aligned}
$$

Since $\tau \leq i-1$, from (6.31), (4.1)(i), and (4.1)(ii) we deduce

$$
A_{n}<c_{p}^{(n-3) / 2}\left|P_{i-1}(\xi)\right|^{-1 / 2}\left|P_{i}\right|^{-1+n / 2}\left|P_{\tau}\right|^{-n+2},
$$

hence by (6.30) and (4.3) we get

$$
\left|\widetilde{P}_{1}\right| \leq A_{2} \leq \ldots \leq A_{n} \leq c_{p}^{(n-3) / 2}\left|P_{i-1}(\xi)\right|^{-1 / 2}\left|P_{i}\right|^{-1+n / 2}\left|P_{\tau}\right|^{-n+2} .
$$


Thus, $A_{1}, \ldots, A_{n}$ satisfy the conditions of Statement 6.3 . Hence there exists a nonzero polynomial $L(x)=c_{n} x^{n}+\ldots+c_{1} x+c_{0}$ with integer coefficients which satisfies

$$
\begin{gathered}
|L(\xi)|<A_{1} \leq c_{p}^{-1}\left|P_{\tau-1}(\xi)\right|, \\
\left|c_{j_{\nu}}\right| \leq c_{p}^{-1}\left|Q_{\tau}^{(\nu-1)}\right| \quad(\nu=1, \ldots, n-1), \\
|L|<\xi^{-n+1} A_{n}=\xi^{-n+1} c_{p}^{-1}\left|Q_{\tau}^{(n-2)}\right|<\left|Q_{\tau}^{(n-2)}\right| \leq Q_{\tau}^{(n-1)} \mid,
\end{gathered}
$$

where $j_{1}, \ldots, j_{n-1}$ are the indices of the $Q_{\tau}$-system. We obtain a contradiction with the minimality property of $Q_{\tau}^{(n-1)}(x)$. This contradiction proves Corollary 6.4.

7. Proof of the Theorem. We consider a sequence of natural numbers $1=m_{1}<m_{2}<\ldots$ such that

$$
\left|P_{m_{k+1}} \leq \max \left(c_{h}\left|P_{m_{k}},\right| P_{m_{k}+1}\right)<\right| P_{m_{k+1}+1} .
$$

We have

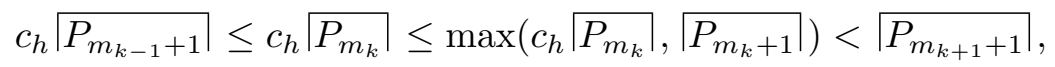

hence

$$
\left|P_{m_{k+1}+1}\right|^{-1}<c_{h}^{-1}\left|P_{m_{k-1}+1}\right|^{-1},
$$

for any natural $k>1$. If we multiply these inequalities together for all $1<k \leq l$, we obtain

$$
{\mid P_{m_{l+1}+1}}^{-1}<c_{h}^{-l / 2}\left|P_{2}\right|^{-1},
$$

where $l$ is even. It follows from Corollary 6.4 that for any $k \in \mathbb{N}$,

$$
\left|P_{m_{k}}(\xi)\right| \prod_{\nu=0}^{n-2}\left|Q_{m_{k}+1}^{(\nu)}\right|<n ! c_{p}^{n}\left|P_{m_{k+1}}(\xi)\right| \prod_{\nu=0}^{n-2}\left|Q_{m_{k+1}+1}^{(\nu)}\right| .
$$

If we multiply these inequalities together for all $1 \leq k \leq l$, we obtain

$$
\left|P_{1}(\xi)\right| \prod_{\nu=0}^{n-2}\left|Q_{2}^{(\nu)}\right|<\left(n ! c_{p}^{n}\right)^{l}\left|P_{m_{l+1}}(\xi)\right| \prod_{\nu=0}^{n-2}\left|Q_{m_{l+1}+1}^{(\nu)}\right|,
$$

for any $l \in \mathbb{N}$. Hence

$$
\left|P_{1}(\xi)\right|<\left(n ! c_{p}^{n}\right)^{l}\left|P_{m_{l+1}}(\xi)\right| \prod_{\nu=0}^{n-2}\left|Q_{m_{l+1}+1}^{(\nu)}\right| .
$$

Let $l$ be even. We substitute (5.15) into (7.2) and apply first (5.9)(II), then 
(7.1) and the definition of $c_{h}$ :

$$
\begin{aligned}
\left|P_{1}(\xi)\right| & <\left(n ! c_{p}^{n}\right)^{l}\left|P_{m_{l+1}}(\xi)\right|^{1 / 2}\left|P_{m_{l+1}+1}\right|^{-1+n / 2}<\left(n ! c_{p}^{n}\right)^{l} \mid{\mid P_{m_{l+1}+1}}^{-1} \\
& <\left(n ! c_{p}^{n}\right)^{l} c_{h}^{-l / 2}\left|P_{2}\right|^{-1}<\left(n ! c_{p}^{n}\right)^{l} c_{h}^{-l / 2}=\left(\frac{1}{2}\right)^{l} .
\end{aligned}
$$

Letting $l \rightarrow \infty$ we come to a contradiction with the boundedness of $\left|P_{1}(\xi)\right|$. Thus, the assumption

$$
\begin{gathered}
\exists \widetilde{H}_{0}>0 \forall Q(x) \in \mathbb{Z}[x], \operatorname{deg} Q(x) \leq n,|Q|>\widetilde{H}_{0}, \\
\frac{|Q(\xi)|}{\left|Q^{\prime}(\xi)\right|}>c_{T}|Q|^{-A},
\end{gathered}
$$

cannot be true. So neither can (2.1). Hence for any real number $0<\xi<1 / 4$ which is not an algebraic number of degree $\leq n$, we have

$$
\exists c>0 \forall \widetilde{H}_{0}>0 \exists \alpha \in \mathbb{A}_{n}, H(\alpha)>\widetilde{H}_{0}, \quad|\xi-\alpha| \leq c H(\alpha)^{-A},
$$

and this completes the proof of the Theorem.

Acknowledgements. I would like to express my sincere thanks to Prof. V. Bernik for introducing me to this problem. Also, I am very grateful to the referee for his valuable suggestions and useful comments.

\section{References}

[1] V. I. Bernik and K. I. Tishchenko, Integral polynomials with an overfall of the coefficient values and Wirsing's problem, Dokl. Akad. Nauk Belarusi 37 (1993), 9-11 (in Russian).

[2] E. Bombieri and J. Mueller, Remarks on the approximation to an algebraic number by algebraic numbers, Michigan Math. J. 33 (1986), 83-93.

[3] H. Davenport and W. M. Schmidt, Approximation to real numbers by quadratic irrationals, Acta Arith. 13 (1967), 169-176.

[4] A. O. Gelfond, On the algebraic independence of transcendental numbers of certain classes, Uspekhi Mat. Nauk (N.S.) 4 (1949), no. 5 (33), 14-48.

[5] W. M. Schmidt, Diophantine Approximation, Berlin, 1980, 299 p.

[6] V. G. Sprindžuk, A proof of Mahler's conjecture on the measure of the set of S-numbers, Izv. Akad. Nauk SSSR Ser. Mat. 29 (1965), no. 2, 379-436 (in Russian).

[7] K. I. Tishchenko, Systems of linearly independent polynomials with height gaps and Wirsing's problem, Vestsi Akad. Navuk Belarusi Ser. Fiz.-Mat. Navuk 4 (1996), 16-22 (in Russian).

[8] -, On approximation to real numbers by algebraic numbers of the third degree, in: Collection of articles dedicated to the 60th birthday of V. G. Sprindžuk, 1997, 85-96 (in Russian). 
[9] E. Wirsing, Approximation mit algebraischen Zahlen beschränkten Grades, J. Reine Angew. Math. 206 (1961), 67-77.

Institute of Mathematics

Academy of Sciences of Belarus

220072, Surganova 11, Minsk

Belarus

E-mail: ktishchenko@bspa.unibel.by

Received on 10.6.1998

and in revised form on 14.12.1999 